\title{
The Anatomy of Object Recognition-Visual Form Agnosia Caused by Medial Occipitotemporal Stroke
}

\author{
Hans-Otto Karnath, Johannes Rüter, André Mandler, and Marc Himmelbach \\ Section of Neuropsychology, Center of Neurology, Hertie Institute for Clinical Brain Research, University of Tübingen, D-72076 Tübingen, Germany
}

The influential model on visual information processing by Milner and Goodale (1995) has suggested a dissociation between action- and perception-related processing in a dorsal versus ventral stream projection. It was inspired substantially by the observation of a double dissociation of disturbed visual action versus perception in patients with optic ataxia on the one hand and patients with visual form agnosia (VFA) on the other. Unfortunately, almost all cases with VFA reported so far suffered from inhalational intoxication, the majority with carbon monoxide (CO). Since $\mathrm{CO}$ induces a diffuse and widespread pattern of neuronal and white matter damage throughout the whole brain, precise conclusions from these patients with VFA on the selective role of ventral stream structures for shape and orientation perception were difficult. Here, we report patient J.S., who demonstrated VFA after a well circumscribed brain lesion due to stroke etiology. Like the famous patient D.F. with VFA after CO intoxication studied by Milner, Goodale, and coworkers (Goodale et al., 1991, 1994; Milner et al., 1991; Servos et al., 1995; Mon-Williams et al., 2001a,b; Wann et al., 2001; Westwood et al., 2002; McIntosh et al., 2004; Schenk and Milner, 2006), J.S. showed an obvious dissociation between disturbed visual perception of shape and orientation information on the one side and preserved visuomotor abilities based on the same information on the other. In both hemispheres, damage primarily affected the fusiform and the lingual gyri as well as the adjacent posterior cingulate gyrus. We conclude that these medial structures of the ventral occipitotemporal cortex are integral for the normal flow of shape and of contour information into the ventral stream system allowing to recognize objects.

\section{Introduction}

Based on a body of behavioral and anatomical studies, Ungerleider and Mishkin (1982) suggested the existence of a ventral occipitotemporal "what" pathway and of a dorsal occipitoparietal "where" pathway of visual information processing. Milner and Goodale (1995) modified the dual-stream model by suggesting a dissociation between action- and perception-related visual processing in these pathways. This model was inspired substantially by the observation of a double dissociation of disturbed visual action versus perception in neurological patients. While patients with so-called "optic ataxia" show gross misreaching when they point to or grasp objects, they seem to be undisturbed in recognizing and perceiving orientation and shape of these objects. $\mathrm{Pa}$ tients suffering from visual form agnosia (VFA) show the opposite dissociation. While visually guided reaching is preserved, they lost the ability to discriminate between simple geometric shapes and orientation as well as to recognize objects, despite the fact that basic visual abilities like contrast, color, or motion perception remained largely intact.

The location of the brain lesion in the first group of subjects supports the dual-stream concept. Disturbed action-related vi-

Received 0ct. 28, 2008; revised Feb. 24, 2009; accepted March 18, 2009.

This work was supported by the Bundesministerium für Bildung und Forschung (BMBF-Verbundprojekt "Räumliche Orientierung" 01GW0641), the Deutsche Forschungsgemeinschaft (SFB 550-A4), and the European Commission (ERC StG 211078).

Correspondence should be addressed to Prof. Hans-Otto Karnath, Center of Neurology, University of Tübingen, Hoppe-Seyler-Strasse 3, D-72076 Tübingen, Germany. E-mail: karnath@uni-tuebingen.de.

D01:10.1523/JNEUROSCI.5192-08.2009

Copyright $\odot 2009$ Society for Neuroscience $\quad$ 0270-6474/09/295854-09\$15.00/0 sual processing in patients with optic ataxia typically is associated with damage of the parieto-occipital junction (POJ) (Karnath and Perenin, 2005), i.e., with damage located right within the dorsal visual stream. However, the anatomical counterpart inherent in the dual-stream concept (Milner and Goodale, 1995), namely that the severe visual deficits in patients with VFA are the result from damage to ventral stream structures, still is not that clearly documented. The reason is as follows. A review of the very few patients reported in the literature with a clearly documented VFA reveals that with two exceptions (subject $\mathrm{X}$ with inorganic mercury intoxication [Landis et al., 1982] and subject 006 after surgery of an oligodendroglioma [Barton et al., 2004]), all other subjects suffered from diffuse brain damage due to carbon monoxide (CO) intoxication: H.C. (Adler, 1944, 1950), M.D.S. (Benson and Greenberg, 1968, 1969; Efron, 1968), R.C. (Abadi et al., 1981; Campion and Latto, 1985), and patient D.F., who was extensively studied by Milner, Goodale, and other researchers (Goodale et al., 1991, 1994; Milner et al., 1991; Servos et al., 1995; Mon-Williams et al., 2001a,b; Wann et al., 2001; Westwood et al., 2002; McIntosh et al., 2004; Schenk and Milner, 2006). Although imaging of the brains of these patients showed certain regions more affected than others, the effect of CO intoxication is a diffuse and widespread loss of neural tissue throughout the whole brain. Histological postmortem studies as well as structural MRI of human brains after CO intoxication have revealed widespread diffuse degeneration bilaterally of the gray and white matter. Conclusions about the possible role of (particular) ventral stream areas for shape and orientation perception drawn from VFA patients with CO intoxication thus are difficult. It is possible that 
the severe perceptual disorder in these patients did not result from selective dysfunction of only a particular area, e.g., from the damage in occipital and ventral stream structures. Rather, it could be the result of this damage in combination with the complex and widespread pattern of neuronal and white matter damage throughout the whole brain, or even of only the latter.

A better model than CO intoxication to study the role of circumscribed brain areas is stroke. To our knowledge we here present the first case with empirically documented defects in discriminating between simple geometric shapes and orientation as well as in object recognition, resulting from an acute stroke lesion.

\section{Materials and Methods Patient J.S.}

Two months before the present investigation, J.S., a 74-year-old, righthanded former technical engineer, suffered from an ischemic stroke during a coronary angiography. The patient complained about an inability to "see" objects, watch TV, or read newspapers. Dressing as well as other activities of daily living could only be carried out on his own when he knew exactly beforehand where respective objects, e.g., clothing, were located in the room. Otherwise he was unable to visually identify and thus find the wanted items. Identification of objects by their characteristic smell or taste was possible. J.S. also reported difficulties in recognizing familiar people by their face. Even relatives and close friends he could only identify by their voice. In apparent contrast to these severe visual impairments, he could move around safely in his neighborhood. For example, he took regular walks or went to the grocery store for daily shopping (using a written list handed over to the shop assistants).

Ophthalmological investigation revealed no disorders of the peripheral visual system. Clinical neurological testing revealed no paresis, no sensory disorders, and no visual field deficits. Testing for optic ataxia was without abnormalities. The latter was assessed by two investigators, one sitting in front of, the other standing behind the patient. J.S. fixated the nose of the investigator sitting in front of him and grasped a target object that was presented by the second investigator at various locations in the left and right visual half field. Ten reaches were tested for both hands separately. No misreaches were observed.

Neuropsychological testing revealed severely impaired identification of objects. J.S. recognized only 3 of 12 real objects (cup, plate, lemon, lighter, light bulb, fork, apple, knife, box of matches, spoon, pen, tooth brush) presented without time constraint. Haptic information acquired by free tactile exploration of the visually unrecognized objects led to correct identification in 5 objects. In the Fragmented Picture Test [Fragmentierter Bildertest (Kessler et al., 1993)], J.S. failed to recognize any of the fragmented or any of the complete line drawings, both presented without time limit. In the Boston Naming Test from the CERAD test battery (Morris et al., 1989; Satzger et al., 2001), J.S. could identify only 2 of 15 line drawings of real objects. Copying of geometrical line drawings (circle, square, triangle) was correct, but copying simple line drawings of real objects (e.g., comb, racket) was severely disturbed. Identification of colors was largely preserved; J.S. only made 2 errors in 10 presentations of 5 different colors (green, yellow, red, white, blue) in random order. Writing of sentences was without error, while reading his own as well as others' writings was hardly possible. Verbal fluency [from the CERAD test battery (Morris et al., 1989; Satzger et al., 2001)] and number repetition [from the Nürnberger-Alters-Inventar (Oswald and Fleischmann, 1997)] were normal. In apparent contrast to his severe disturbance of visual recognition, J.S. moved around safely during the inpatient period in the various rooms of our clinic and never collided with potential obstacles. He was able to shake hands adequately, and looked straight in the face of the examiner as well as at objects on the table he was asked to manipulate. J.S. grasped these objects with quick, smooth, and appropriate movements.

\section{Experimental examination}

The experimental investigation was designed to test for a possible dissociation of action and perception in the present stroke patient J.S. that could be comparable to the behavioral profile of the well known patient, D.F., who suffered from VFA due to CO intoxication (Goodale et al., 1991; Milner et al., 1991). We chose patient D.F. from the group of reported cases with VFA because her pattern of preserved and disturbed abilities is extremely well documented and because some of the most compelling evidence for the perception versus action hypothesis has come from this patient. Among the various tasks that were used to compare action- with perception-related processing in D.F., we selected three prototypical experiments for the examination of patient J.S. These were as follows: perceptual orientation matching versus posting of a plaque during reaching (Goodale et al., 1991; Milner et al., 1991), size estimation versus grasping of rectangular objects (Goodale et al., 1991), and perceptual discrimination versus grasping of irregularly shaped objects (Goodale et al., 1994). We used an optoelectronic motion analyzer (BTS Elite) for movement recordings in experiments 1 and 2 (see below). Each recording was acquired with a sampling frequency of $100 \mathrm{~Hz}$. Data analysis was performed off-line. Three-dimensional time-position data of all individual markers were filtered before further analysis, applying the automatic model-based bandwidth selection procedure LAMBDA (D'Amico and Ferrigno, 1992).

Apart from patient J.S. we examined nine right-handed control subjects (mean age: 75 years, range $70-81,4$ females). All control subjects had normal or corrected to normal vision and had no recorded history of neurological or psychiatric disorders. We tested for behavioral dissociations in J.S. using the Revised Standardized Difference Test (RSDT) suggested by Crawford and Garthwaite (2005), comparing the discrepancy between J.S.'s performance in the respective visuomotor and perceptive tasks with the discrepancies in the group of controls. We further tested whether his performance in single tasks was significantly different from healthy controls using revised $t$ tests as suggested by Crawford and Howell (1998). All individuals gave their informed consent to participate in the study that has been performed in accordance with the ethical standards laid down in the 1964 Declaration of Helsinki.

Experiment 1: perceptual orientation matching versus posting of a plaque during reaching. According to the original experiment in D.F. (Milner et al., 1991), a black disk (diameter $250 \mathrm{~mm}$ ) with an oblong slot (width 125 $\mathrm{mm}$, height $38 \mathrm{~mm}$ ) was presented. The perceptual and the visuomotor task were conducted blockwise. The subject was sitting at a table. Out of sight of the subject, the slot was turned to a random angle between $+90^{\circ}$ and $-90^{\circ}$ (vertical slot orientation was defined as $0^{\circ}$ ). The target disk then was presented at a distance of $400 \mathrm{~mm}$ in front of the subject at a height of $\sim 300 \mathrm{~mm}$ above the table.

In the perceptual condition, the subject was instructed to manually rotate a second identical disk in such a way, that the orientation of the second slot was in agreement with the target slot's orientation (Fig. 1A). Two infrared markers were attached to each disk in alignment with the respective slot. The subject held the response disk in front of his body at about the same height as the target disk, rotated it, and indicated verbally when he had accomplished the final estimate of the target's orientation. Upon this signal, target and response orientations were recorded with the BTS motion analyzer. In the visuomotor condition, the subject was asked to insert an oblong plastic card $(180 \times 70 \times 3 \mathrm{~mm})$ into the slot of the target disk (Fig. $1 B$ ). In addition to the infrared markers on the target disc, two markers were attached to the corners of the plastic card facing the target disk. At the beginning of each trial the hand with the card rested at a home position at the table top $\sim 100 \mathrm{~mm}$ anterior to the subject's chest. Hand movements and motion recordings started upon a go signal from the experimenter. For each trial we determined the shortest positive distance of the card's front edge to the disk's surface, i.e., the sample just before the insertion of the card. Second, we calculated the deviation between the card's orientation at this moment and the orientation of the target slot.

Experiment 2: size estimation versus grasping of rectangular objects. Three square-shaped, gray-colored objects $\left(40 \times 40 \times 20 \mathrm{~mm}^{3}, 60 \times\right.$ $60 \times 20 \mathrm{~mm}^{3}, 80 \times 80 \times 20 \mathrm{~mm}^{3}$ ) were presented to the subject. As in the original study in D.F. (Goodale et al., 1991) the experiment consisted of a perceptual and a visuomotor task. The tasks were conducted blockwise starting with the perceptual task. The subject was sitting at a table with the right hand resting at a home position $100 \mathrm{~mm}$ in front of him. In 
both conditions, one of the objects then was presented on the table at a distance of $400 \mathrm{~mm}$ anterior to the subject's chest. Object and home positions were aligned with the subject's body midline. Two infrared markers were attached to the finger tips for motion capturing. In the perceptual condition, the subject was instructed to indicate the size of the respective object at the home position using his index finger and thumb without any transport movement toward the objects. After the subject indicated verbally that he reached the final estimate of the object's size, the experimenter recorded the finger positions with the BTS motion analyzer. We calculated the mean finger aperture over a recording time of $1 \mathrm{~s}$ for the analysis of the perceptual data. In the visuomotor condition, the subject actually picked up the object with index finger and thumb. His movement started upon a go signal from the experimenter who started kinematic acquisitions simultaneously. We determined the maximum grip aperture (MGA) that occurred during the grasping movement.

In addition, kinematic variables were calculated to further describe J.S.'s movements compared with controls. As we were primarily interested in MGA, we recorded no reaction times and tracked no additional wrist markers. Movement time (MT) was therefore determined by identification of the second finger marker whose velocity exceeded $50 \mathrm{~mm} / \mathrm{s}$ and the first finger marker whose velocity fell below 50 $\mathrm{mm} / \mathrm{s}$. Furthermore, we calculated the mean of both fingers' peak velocity (PV). Movement time of each movement was standardized to a length of 100 samples to allow for a comparison of the relative timing of each movement. We calculated the relative time of the occurrence of MGA (\%TMGA) and PV (\%TPV).

Experiment 3: perceptual discrimination versus grasping of irregularly shaped objects. Equivalent to the experiment in D.F. conducted by Goodale et al. (1994), we compared the ability of J.S. to discriminate objects of different shape with his ability to position his fingers correctly on the boundaries of the same objects when he was asked to pick them up. Twelve different shapes (two of each) were used (see Fig. $4 A$ ). The plastic shapes were $0.5 \mathrm{~cm}$ thick, were painted uniformly gray and were presented on a flat black background. Their size was between 2 and $5 \mathrm{~cm}$ in any direction of the horizontal plane. The subject was seated at a table on which a pair of shapes was placed behind a black cover. In each trial, two identical or two different shapes were presented. The relative orientation of the two shapes varied from trial to trial, with the principal axis of one shape being rotated $0^{\circ}$, $90^{\circ}, 180^{\circ}$, or $270^{\circ}$ with respect to the other. Each trial consisted of two parts: a perceptual same/different discrimination task and a grasping task. At the beginning of each trial a black cover prevented the subject from seeing the objects. Behind the cover, two shapes were placed at a distance of 20 and $30 \mathrm{~cm}$ to the subject's chest along the body midline. The shapes were placed along a vertical line to avoid any potential confounds between location and half field of view. Each trial started by removing the black cover. During or after an initial presentation time of $3 \mathrm{~s}$ the subject reported whether the shapes were the same or different. After this response, the shapes were covered again and the experimenter removed one of the two shapes. The cover was removed again and the subject picked up the remaining shape by using his thumb and index

\section{B) Action}

\section{A) Perception}
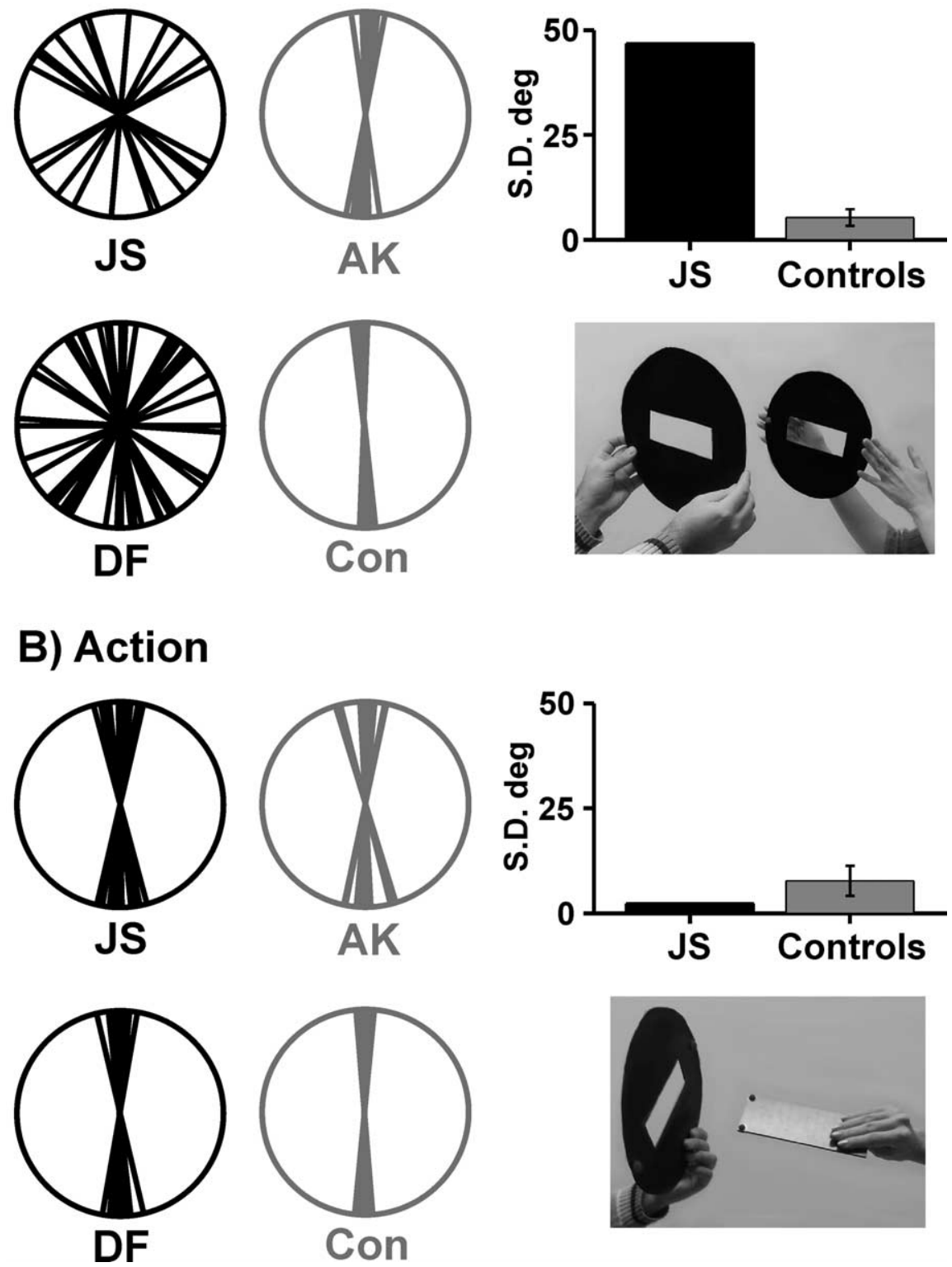

Figure 1. Polar plots illustrating perceptual orientation judgements $(\boldsymbol{A})$ and orientation adaptation in reaching movements vertical. The polar plots therefore show difference values to the vertical, representing a difference to the target orientation of $0^{\circ}$. Black data plots indicate the data of our patient J.S. and the data of VFA patient D.F. reported by Milner and Goodale (1995). Gray polar plots indicate an exemplary control of our study (A.K.) and the control subject reported by Milner and Goodale (1995) (Con). Bar plots illustrate SDs of J.S.'s responses in either task and average SDs in our group of healthy controls (error bars denote 1 SD).

finger. Each subject received 5 practice trials, followed by 96 experimental trials, 48 trials with same shapes and 48 trials with different shapes. Each shape was presented 16 times in a pseudorandomized sequence, 4 times in "same" trials and 8 times in "different" trials. A minimum of 6 grasping movements were successfully recorded for each shape in each subject. The relative orientation was varied randomly with each relative orientation of a shape presented at least once.

The grasping movements were recorded using a MiniDV-Camcorder (DCR-HC14E, Sony Corporation) that was affixed $\sim 70 \mathrm{~cm}$ above the table providing a free and orthogonal view on the objects and the moving hand. In a frame-by-frame analysis we determined the time of first contact between the fingers and the respective shape. Subsequently we determined the center positions of the two contact points manually. We drew a line between these positions (grasp line) and calculated its perpendic- 


\section{A) Perception}

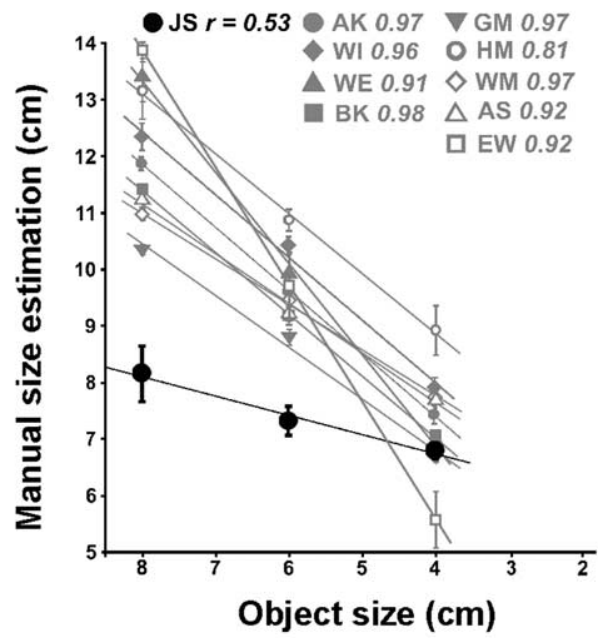

\section{B) Action}
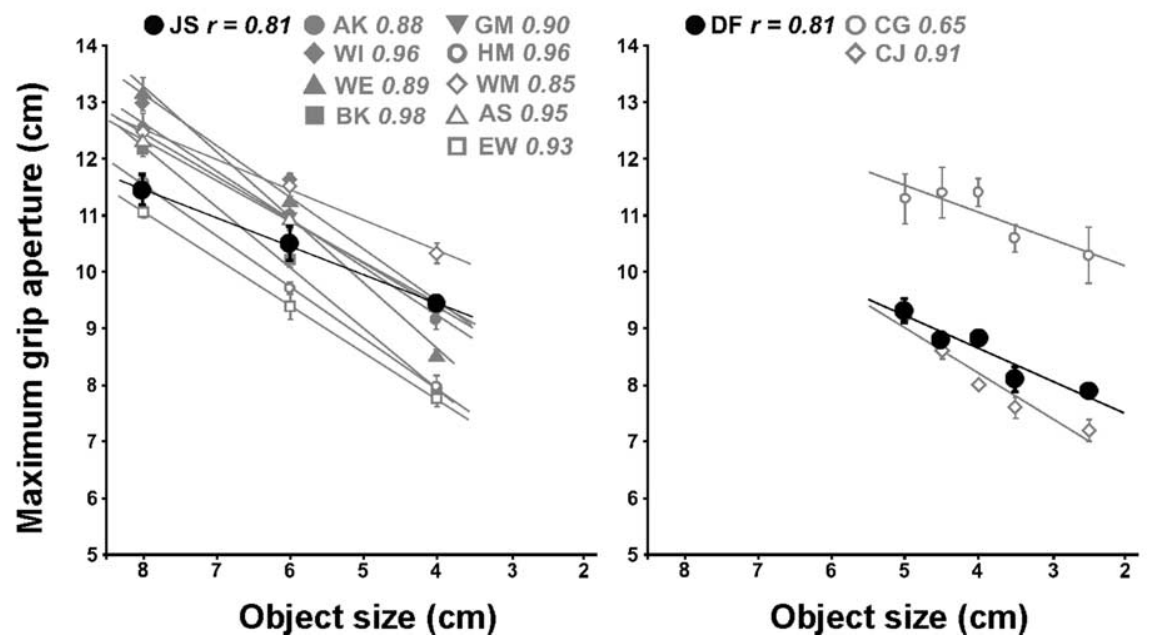

Figure 2. Manual size estimation (MSE) for rectangular blocks of different size ( $\boldsymbol{A})$ and maximum grip aperture (MGA) during grasping of the same objects $(\boldsymbol{B})$. All plots have been adjusted to the same spatial scale to allow for a valid comparison of the individual scaling of finger aperture on object size. Please note that scaling of MSE and MGA on various object sizes rather than absolute measures of MSE and MGA are of importance here. Left panels, Mean data (with SE) of our patient J.S. (black symbols) and nine control subjects (gray symbols). Right panels, Mean data (with SE) of VFA patient D.F. (black symbols) and two control subjects (C.G., C.J.: gray symbols) taken from Goodale et al. (1991). Correlation coefficients for each subject are given above each plot, while the lines represent linear regressions of MSE or MGA, respectively, on object size.

ular distance from the center of mass of the respective shape (compare Fig. 4C). As in the original experiment (Goodale et al., 1994) this measure was taken to quantify the subject's performance. It reflects the subject's processing of the overall shape of an object while grasping, as it is known that in healthy subjects the grasp line typically passes through the center of mass (Iberall et al., 1986; Blake and Brady, 1992).

\section{Imaging}

MR scans were obtained on a $1.5 \mathrm{~T}$ echoplanar imaging (EPI) capable system (Magnetom Sonata, Siemens) $10 \mathrm{~d}$ after stroke onset. Fluidattenuated inversion-recovery (FLAIR) imaging was used for the depiction of lesioned brain tissue. FLAIR represents a T2-weighted imaging protocol which suppresses the signal of the CSF and provides high sensitivity for acute and subacute infarcts (Brant-Zawadzki et al., 1996; Noguchi et al., 1997; Ricci et al., 1999). The FLAIR sequence comprised 24 coronal slices, a field of view (FOV) of $186 \times 230 \mathrm{~mm}^{2}$, a $207 \times 256$ matrix, a repetition time (TR) of $9000 \mathrm{~ms}$, and an echo time (TE) of $118 \mathrm{~ms}$.

\section{Results}

Experiment 1: perceptual orientation matching versus posting of a plaque during reaching

In the perceptual condition, J.S. demonstrated a large variability in his orientation judgements compared with both healthy controls (Fig. 1A). The SD of J.S.'s judgements was $46.8^{\circ}$ in striking contrast to the very accurate performance of the control group (mean of individual SDs: $5.3^{\circ}$, SD $\left.2.1^{\circ} ; t_{(8)}=18.748, p_{(\text {one-tailed })}=0.000001\right)$. SDs of our data are comparable to the reported quantitative data of D.F.'s orientation judgements. Milner et al. (1991) (their Fig. 11) reported SDs from $46.8^{\circ}$ to $60.45^{\circ}$ for D.F. and values between $4.83^{\circ}$ and $13.23^{\circ}$ for two healthy controls. In contrast to this severe perceptual deficit, J.S. demonstrated smooth adaptations of the orientation of his hand to the orientation of the target slot when posting a plaque into the slot during a reaching movement (Fig. 1B). All his movements were brisk and uninterrupted. The SD of his responses was $9.0^{\circ}$. This dispersion was comparable to the variability of responses in the control group (mean SD: $7.7^{\circ}, \mathrm{SD}$ $\left.3.7^{\circ} ; t_{(8)}=0.333, p_{(\text {one-tailed })}=0.374\right)$. The analysis of a dissociation between perceptual orientation matching and posting of a plaque during reaching showed a considerably higher discrepancy between the tasks in J.S. compared with controls $\left(t_{(8)}=\right.$ $\left.13.829, p_{\text {(two-tailed) }}<0.00001\right)$.

\section{Experiment 2: size estimation versus grasping of rectangular objects} Estimating the size of the presented objects J.S. demonstrated some correlation between finger aperture and object size $(r=$ 0.53) (Fig. $2 A$ ). However, his performance was considerably different from controls (mean $r=0.93$, SD $0.05 ; t_{(8)}=-7.779$, $\left.p_{\text {(one-tailed) }}=0.00003\right)$. In contrast, if J.S. actually grasped the same objects, his MGA was much better related to object size $(r=0.81)$. Although being slightly impaired in this task as well, his performance was closer to the behavior of healthy subjects (mean $r=0.92$, SD $0.04 ; t_{(8)}=$ $-2.609, p_{\text {(one-tailed) }}=0.016$ ) (Fig. $2 B$ ). The analysis of a dissociation between size estimation and grasping of rectangular objects showed a considerably higher discrepancy between the tasks in J.S. compared with controls $\left(t_{(8)}=3.033, p_{\text {(two-tailed) }}=0.016\right)$. This behavioral dissociation in J.S. is again comparable to D.F.'s data. Goodale et al. (1991) reported a correlation of $r=0.10$ for size estimation in D.F. (control subjects 1 and 2: $r=0.82, r=$ 0.65 ) and a correlation of $r=0.81$ (control subjects 1 and 2: $r=$ $0.65, r=0.91$ ) if D.F. actually grasped the objects (see also Fig. 2).

After standardization of each individual aperture trajectory we calculated mean aperture time courses for each participant and subsequently a group mean aperture time course for the 
control group. Figure 3 illustrates that J.S.'s aperture was within a region of one SD around the healthy group's mean aperture most of the time and always within a region of two SDs. Nevertheless, the standardized trajectories underline the abovementioned finding of a smaller correlation between object size and MGA in J.S. compared with healthy controls. Furthermore, J.S. seemed to produce MGA earlier than controls. Indeed, the difference between J.S. and controls in \%TMGA approached significance (J.S.: 60\%; controls: mean $79 \%$, SD $8 \% ; t_{(8)}=-2.253, p_{\text {(two-tailed) }}=$ 0.054). Analyses of PV (J.S.: $1453 \mathrm{~mm} / \mathrm{s}$; controls: $918 \mathrm{~mm} / \mathrm{s}, \mathrm{SD} 253 \mathrm{~mm} / \mathrm{s} ; t_{(8)}=$ 2.006, $p_{\text {(two-tailed) }}=0.080$ ), MT (J.S.: 597 ms; controls: $813 \mathrm{~ms}$, SD $133 \mathrm{~ms} ; t_{(8)}=$ $\left.-1.541, p_{\text {(two-tailed) }}=0.162\right)$, and $\% \mathrm{TPV}$ (J.S.: 29\%; controls: 38\%, SD 7\%; $t_{(8)}=$ $\left.-1.220, p_{\text {(two-tailed) }}=0.257\right)$ clearly exceeded the statistical threshold.

\section{Experiment 3: perceptual discrimination versus grasping of irregularly shaped objects}

Figure $4 B$ illustrates that J.S.'s perceptual discrimination abilities were clearly inferior to healthy controls $t_{(8)}=-7.589, p_{\text {(one-tailed) }}$ $=0.00003)$. Although his performance was above chance level with $73 \%$ correct responses, the control subjects were almost perfect in the same task (mean: 97\%, SD 3\%). In contrast, much like D.F., J.S.'s visuomotor performance was comparable to healthy controls. Grasp lines were consistently oriented along primary axes of the shapes with grasp points located on regions of maximum convexity or concavity (Fig. 4C). The quantitative analysis of the distances between grasp lines and the center of mass of the shapes revealed slightly worse performance in J.S. compared with controls (J.S.: mean $2.52 \mathrm{~mm}$, SD 2.65; controls: mean 1.9, SD 0.2). Albeit this difference in visuomotor accuracy between J.S. and controls was significant $\left(t_{(8)}=2.846, p_{\text {(one-tailed) }}\right.$ $=0.0108)$, the discrepancy between perceptual discrimination and grasping of irregularly shaped objects was much larger in J.S. than in controls $\left(t_{(8)}=6.4, p_{\text {(two-tailed) }}=0.00021\right)$. Unfortunately, Goodale et al. (1994) reported no individual values for this variable in D.F. and controls. Instead, these authors reported frequency distributions of the distances between grasp lines and the respective center of mass for each subject. These distributions suggested a comparable performance of patient D.F. and a healthy control (Goodale et al., 1994) (see also Fig. $4 C$ ). Corresponding histograms of J.S. and group values of our controls underline the behavioral resemblance between D.F. and J.S. (Fig. 4C).

\section{Anatomy}

Fluid-attenuated inversion-recovery MR (FLAIR) imaging revealed a stroke affecting the vascular territory of the posterior cerebral artery bilaterally (Fig. 5). The lesion was constrained to the medial aspects of the ventral occipitotemporal cortex. The lateral cortical structures of the occipital and temporal lobes were spared and structurally intact. Bilaterally the lesion encompassed the fusiform and the lingual gyri, and extended into the isthmus of the adjacent posterior cingulate gyrus. In the right hemisphere, the lesion also extended into the parahippocampal gyrus and into the cuneus.
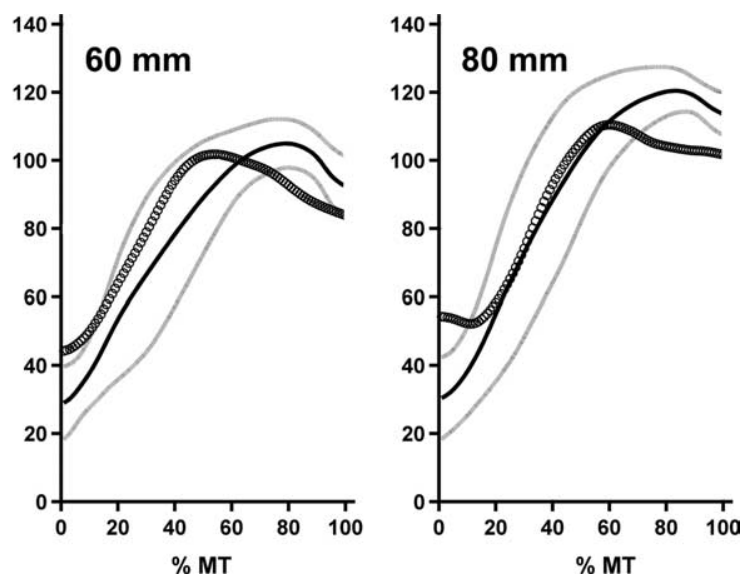

Figure 3. Time courses of mean grip aperture for the three target object sizes ( $40 \mathrm{~mm}, 60 \mathrm{~mm}, 80 \mathrm{~mm})$. The abscissa denotes relative movement time (\% MT) from the kinematically defined movement start (0\%) to movement end (100\%). The ordinate denotes absolute grip aperture. The thick black line represents the mean aperture of the control group with one SD (indicated as gray lines) below and above each mean value. Circles represent our patient J.S.'s mean aperture.

\section{Discussion}

The present results revealed clear evidence for VFA in patient J.S. We found his shape and orientation perception as well as the identification of real objects severely impaired. Perception of other visual qualities such as color or movement were largely intact. Also, J.S.'s reaching for objects was well preserved. Our experimental results were very similar to those observed in the extensively studied patient D.F. with VFA (Goodale et al., 1991, 1994; Milner et al., 1991; Servos et al., 1995; Mon-Williams et al., 2001a,b; Wann et al., 2001; Westwood et al., 2002; McIntosh et al., 2004; Schenk and Milner, 2006). Much like D.F., J.S. showed an obvious dissociation between disturbed visual perception of shape and orientation information on the one hand and preserved visuomotor abilities based on the same information on the other.

Based on this behavioral dissociation, Milner and Goodale (1995) suggested that the brain damage suffered by D.F. as a consequence of her intoxication interrupted the normal flow of shape and contour information into the perceptual, ventral stream system that, if functioning properly, enables us to recognize objects. At the same time the processing of shape and contour information by D.F.'s visuomotor control systems in the dorsal stream was preserved and allowed her to reach out and grasp objects properly.

Based on a first MRI 13 months after CO intoxication, the brain damage of D.F. had been described as a bilateral lesion of the occipital poles, mainly affecting the lateral aspects of the occipital gyri and extending into the posterior parasagittal occipitoparietal region, while leaving primary visual cortex intact (Milner et al., 1991). Beyond, the authors described bilateral damage of the globus pallidus as well as a global cortical atrophy with a widening of ventricles and sulci. On the basis of a more recent structural MR scan it was suggested that damage of specifically the lateral aspects of the occipitotemporal cortex encompassing the lateral occipital complex (LOC), a structure that has been implicated in object recognition (Malach et al., 1995; Kanwisher et al., 1996), might be the critical anatomical location causing D.F.'s deficit (James et al., 2003). James et al. (2003) speculated that with the damage of area LOC patient D.F. lost the visual brain region that is associated with the normal recognition of objects based on contour information and that therefore the form processing network in D.F.'s ventral stream was heavily impaired. 
A) Shapes

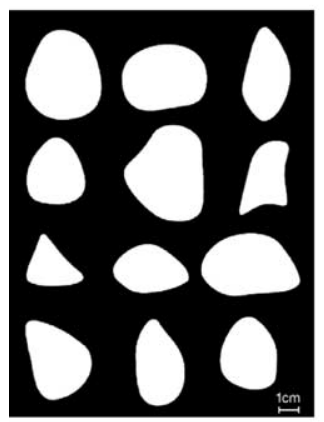

B) Discrimination
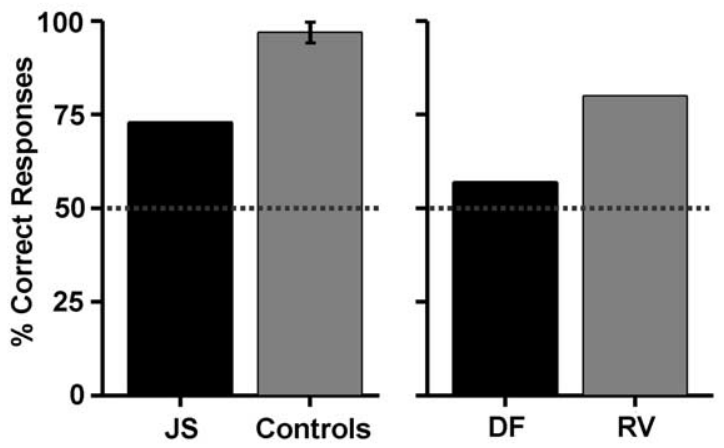

\section{C) Grasping irregular shapes}
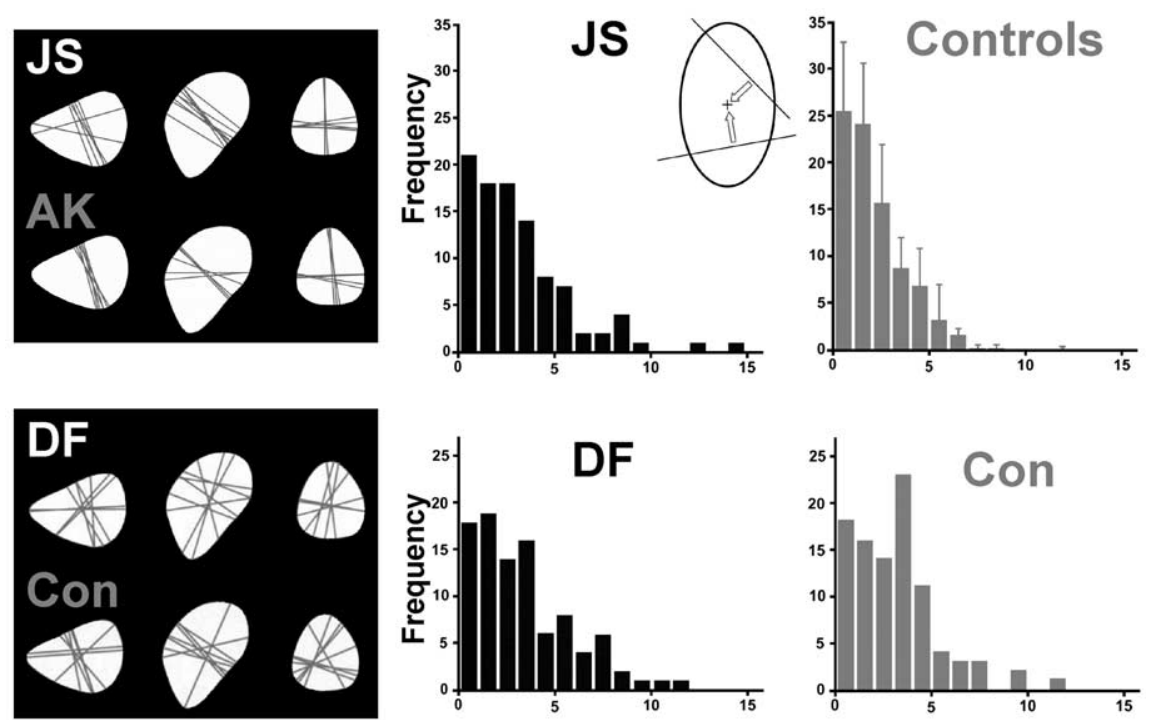

Distance from center of mass $(\mathrm{mm})$

Figure 4. Perceptual discrimination and grasping of objects. $\boldsymbol{A}$, Two sets of 12 irregular shapes were used. $\boldsymbol{B}$, Perceptual discrimination performance between shapes. Left, Performance of our patient J.S. (black) and the average performance (1 SD) of our group of healthy controls (gray). Right, Performance of VFA patient D.F. (black) and a brain-damaged control subject R.V. (gray) taken from the study by Goodale et al. (1994). The brain-damaged control subject R.V. suffered from optic ataxia; a healthy control was not explicitly reported for this task in Goodale et al. (1994). C, Grasping irregular shapes. Data of the current study are shown in the upper row; data of Goodale et al. (1994) are illustrated in the lower row. Grasping lines connecting the contact points at the respective shape are shown for three exemplary shapes for our patient J.S. and an exemplary control of our study (A.K.), as well as for the VFA patient D.F. and a healthy control subject (Con) from Goodale et al. (1994). For a quantitative comparison we analyzed the perpendicular distance between grasp lines and the center of mass across shapes as shown in the inlay. The diagrams show the frequency distributions of these distances for our patient J.S. and the mean distribution for our healthy control group (upper row) with SDs, as well as the data from VFA patient D.F. and a matched control subject (Con) reported by Goodale et al. (1994) (lower row). Bin size of the histograms is $1 \mathrm{~mm}$.

The anatomical findings obtained in the present study suggest a different view. MR imaging of J.S. showed damage of the medial aspect of the ventral occipitotemporal cortex. The bilateral lesion encompassed the fusiform and lingual gyri as well as the isthmus of the adjacent posterior cingulate gyrus. In the right hemisphere, the lesion further extended into the parahippocampal gyrus and into the cuneus. The data thus let us suggest that medial structures rather than the lateral convexity and LOC of the ventral occipitotemporal cortex are integral to the normal flow of shape and contour information into the perceptual, ventral stream system allowing object recognition. Interestingly, this conclusion is supported by the location of the tumor in patient 006 with VFA reported by Barton et al. (2004). This subject underwent neuro- surgery due to an oligodendroglioma and accompanying hemorrhage located in the right medial occipitotemporal cortex. MRI 7 months after surgery showed an extensive right medial occipitotemporal resection, involving the lingual and fusiform gyri. However, due to tumor etiology we do not know, of course, how far the tumor had already invaded into the (on MR scans) apparently healthy looking tissue on the more lateral aspects of the ventral occipitotemporal cortex.

How can we understand the discrepancy between the present anatomical findings and the observations made in D.F.? A very obvious but yet not rejectable explanation could be that both lateral (cf. D.F.) as well as medial (cf. J.S.) structures of the ventral occipitotemporal cortex are integral to the normal flow of shape and contour information into the ventral object recognition system. A further possibility could be that in D.F. the medial structures of the ventral occipitotemporal cortex were not entirely preserved after $\mathrm{CO}$ intoxication. Indeed, although MR imaging of D.F.'s brain showed a relative concentration of damage bilaterally in the ventral LOC (James et al., 2003), histological postmortem studies of human brains after $\mathrm{CO}$ intoxication have revealed that the toxic agent causes widespread diffuse degeneration bilaterally of the gray and white matter. Sections have shown necrosis of gray matter tissue throughout cortex, basal ganglia, hippocampus, and the cerebellum, as well as marked progressive changes of glia cells and multifocal necrotic demyelinization in the white matter affecting intrahemispheric and interhemispheric connections (Hsü and Ch'eng, 1938; Lapresle and Fardeau, 1967). Correspondingly, MRI studies in patients with CO intoxication have revealed diffuse cortical brain atrophy as well as damage in the basal ganglia, caudate nucleus, thalamus, periventricular and subcortical white matter, hippocampus, and cerebellum (Kim et al., 2003; Durak et al., 2005; Lo et al., 2007). In agreement with these findings, the high-resolution MR scans of D.F.'s brain revealed diffuse cortical damage (James et al., 2003). As in the initial investigation by Milner et al. (1991), James et al. (2003) found widespread cortical atrophy with enlarged sulci and ventricles. This loss of neuronal tissue was particularly prominent in the intraparietal sulcus, the postcentral sulcus, the inferior precentral sulcus, and the parieto-occipital sulcus bilaterally, as well as the calcarine sulcus in the left hemisphere. A further MRI lesion was observed in the left posterior parietal cortex. James et al. (2003) as well as later Steeves et al. (2004) speculated that all this widespread brain damage caused by the toxic agent might not be relevant for the deficits of D.F. Rather, they based their conclusions on the relative concentration of bilateral damage in the ventrolateral LOC. 
While this assumption may be true, the authors, however, likewise cannot exclude the alternative possibility, namely that the behavioral deficits in D.F. are not the result of selective dysfunction of only area LOC but rather the consequence of this damage in combination with the widespread pattern of neuronal and white matter damage throughout D.F.'s brain (which-among others-also may have affected the function of medial structures in the ventral occipitotemporal cortex).

As the same argument is true for the other cases with VFA and CO intoxication, what has been reported from these patients with respect to location of brain damage? Images of the lesion of M.D.S. (Benson and Greenberg, 1968, 1969; Efron, 1968) have not been published. Low-resolution CT images of R.C.'s brain revealed diffuse damage with bilateral foci-left more than right-in the striate and prestriate cortex above the calcarine sulci (Abadi et al., 1981; Campion and Latto, 1985). The CT and MRI scans obtained from H.C. 40 years after CO intoxication revealed bilateral occipital atrophy most prominently in subcortical occipital

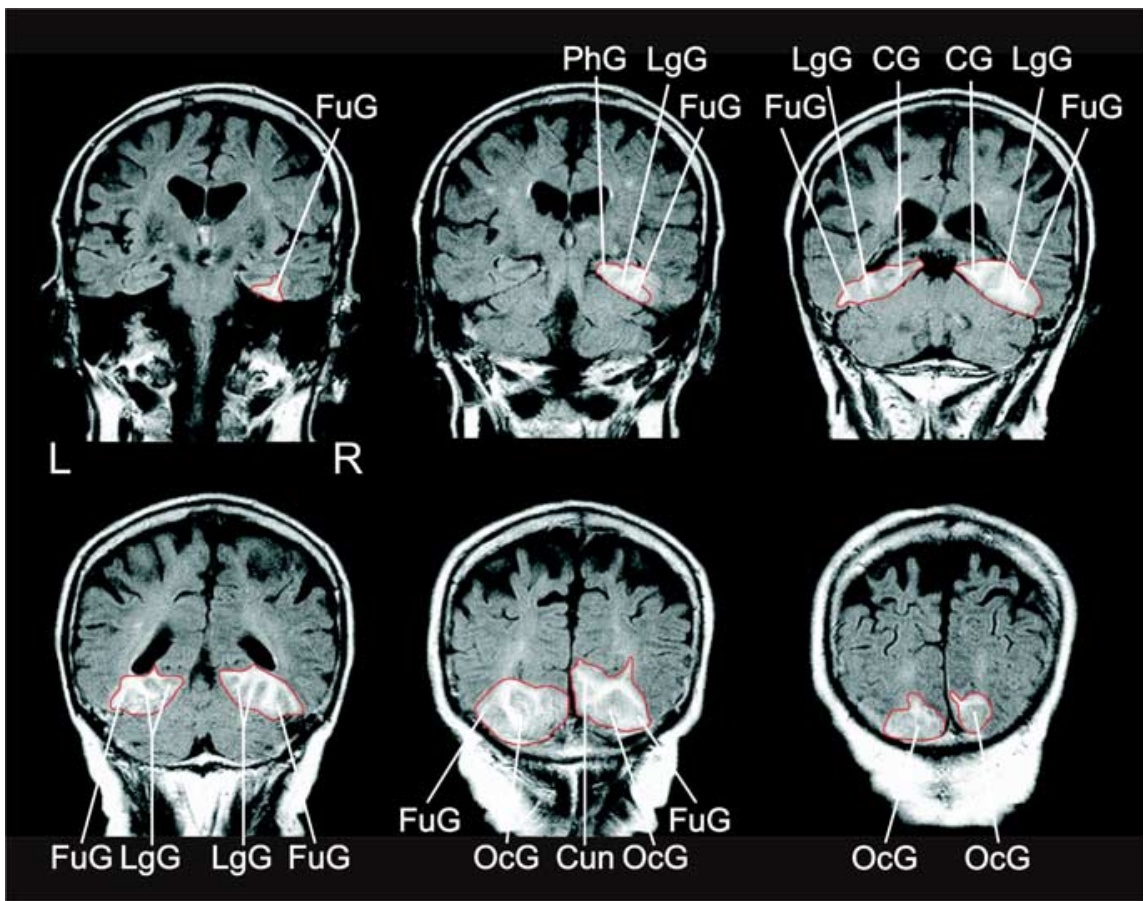

Figure 5. FLAIR magnetic resonance images of the bilateral lesion of our patient J.S. recorded $10 \mathrm{~d}$ after stroke. Coronal sections are shown in neurological convention. The well defined outline of the lesion is emphasized by a red contour. FuG, Fusiform gyrus; $\mathrm{LgG}$, lingual gyrus; PhG, parahippocampal gyrus, $0 \mathrm{CG}$, occipital gyri; $\mathrm{CG}$, cingulate gyrus; Cun, cuneus.

structures, at the occipital convexity, and

the right cuneus (Sparr et al., 1991). CT imaging in the only case with VFA following an intoxication with inorganic mercury (subject X) did not reveal pathological signs (Landis et al., 1982). In conclusion, these different anatomical findings hardly allow firm conclusions that would help solving the apparent discrepancy in anatomy between J.S. and D.F.

Given the lack of diffuse brain damage in the present case J.S., what is known about the cognitive functions represented in the ventromedial occipitotemporal cortex? The fusiform, parahippocampal, and lingual gyri have been at the center of a lively debate on the organization of object representation in the ventral stream (Kanwisher, 2000; Haxby et al., 2001; Levy et al., 2001). One interpretation was that the ventromedial occipitotemporal cortex contains areas, such as the "fusiform face area," the "parahippocampal place area," the "fusiform or extrastriate body area," or the "visual word form area," that are specialized for the representation of specific object categories, such as faces, topographical scenes, human body parts, or words (Puce et al., 1995; Kanwisher et al., 1997; Epstein and Kanwisher, 1998; Downing et al., 2001; McCandliss et al., 2003; Grill-Spector et al., 2004; Peelen and Downing, 2005). In contrast to the concept of categoryspecific representations of objects, Haxby et al. (2001) argued that the ventromedial occipitotemporal cortex encompasses a distributed collection of feature analyzers as part of a broad neural network that represents objects by distributed patterns of connected activation. Alternative proposals suggested that information processing in the medial occipitotemporal areas rather may be understood as an expert system distinguishing visually similar exemplars of any object category for which the viewer has substantial expertise (Gauthier et al., 2000; Tarr and Gauthier, 2000). Further, it was proposed that-in parallel to early visual areas with their retinotopic arrangement-the major principle of organization in higher-order visual areas might be a kind of centerperiphery topography (Levy et al., 2001; Hasson et al., 2002).
The present case with VFA following a bilateral lesion of the ventromedial occipitotemporal cortex cannot help to solve this controversy regarding specific functional characteristics of this area. However, based on the lesion location found in J.S. it can be concluded that the structures in the ventromedial occipitotemporal cortex appear to be indispensable for the normal processing of shape and of contour information within the ventral stream system that allows us to recognize objects.

The two-visual-stream hypothesis of Milner and Goodale (1995) explains the present behavioral and anatomical findings in J.S. very well. From this point of view, our findings can be seen as additional evidence for the reinterpretation of Ungerleider and Mishkin's (1982) model by Milner and Goodale (1995). While Ungerleider and Mishkin's (1982) theory cannot explain the task-specific dissociation of a patient's capabilities to use size and orientation information, Milner and Goodale's (1995) model correctly predicts the pattern of preserved and disturbed abilities observed in J.S. and D.F. However, it also must be said thateven including patient J.S. - the action-perception two-visualstream model still rests on a very small number of patients. Our current knowledge about behavioral dissociations in VFA is almost entirely based on patient D.F. with today 37 original articles and reviews (February 2009). Also, it appears as if the anatomical distinction between perception and action has been overemphasized in the last decade. For example, recent fMRI findings by Konen and Kastner (2008) in healthy subjects suggested the existence of a hierarchical neural system for object information not only in the ventral but also in the dorsal pathway. While in their experiment the dorsal object-related activity appeared indistinguishable from that in the ventral stream, the anatomical findings in patients exhibiting VFA demonstrate that some difference in function must exist between the two systems. Behavioral disturbances to recognize objects and/or to discriminate between simple geometric shapes and orientation are associated with brain 
lesions injuring ventral occipitotemporal, not dorsal occipitoparietal areas. Nevertheless, Konen and Kastner's (2008) findings clearly argue against an oversimplified functional "what"-versus"where" dissociation in relation to ventral versus dorsal stream structures. Further evidence comes from some detailed examinations of D.F., suggesting that her action control was not unimpaired per se. Schenk (2006) observed that D.F.'s performance was impaired in allocentric tasks regardless of whether visuomotor or perceptual processing was required. In contrast, egocentric spatial processing seemed to be spared in D.F. This report was in agreement with previous demonstrations of similar deficits in visuomotor control if allocentric stimulus coding was required from D.F. (Dijkerman et al., 1998; McIntosh et al., 2004). Also in the present investigation, patient J.S. revealed impaired visuomotor performance in two experiments. These relatively small deficits do not invalidate the finding of a behavioral dissociation between perceptual deficits and visuomotor competences in J.S. However, the observation of these impairments in a case with clear-cut VFA after stroke reminds us to be careful in our interpretation of findings in individual VFA patients. J.S.'s small visuomotor impairments could only be elucidated in a statistical comparison to a group of control subjects. Such comparisons have rarely been conducted for other patients with VFA and thus less prominent (visuomotor) deficits might not have been detected in these cases.

Unfortunately, J.S. cannot participate in further experiments and therefore cannot be examined in more detail for sophisticated functional distinctions. Nevertheless, the results presented here support the general idea of a representation of perceptual visual information processing with limited direct connection to human action control. The clear-cut anatomical finding in J.S. suggests that the medial aspect of the ventral occipitotemporal cortex represents a crucial substrate of this processing. This conclusion is supported by the lesion location in the medial parts of the right occipitotemporal cortex following tumor resection in a previous case with VFA (Barton et al., 2004). However, although the present anatomical conclusion is based on a patient thatdue to stroke rather than $\mathrm{CO}$ intoxication or tumor etiologyallows for a more precise localization of brain damage, it must be considered cautiously until further patients with VFA lacking diffuse brain damage have been studied. Alternative conclusions, such that, e.g., both lateral as well as medial structures of the ventral occipitotemporal cortex are integral to the normal flow of shape and contour information into the ventral object recognition system, should not be rejected yet.

\section{References}

Abadi RV, Kulikowski JJ, Meudell P (1981) Visual performance in a case of visual agnosia. In: Functional recovery from brain damage (van Hof MW, Mohn G, eds), pp 275-286. Amsterdam: Elsevier.

Adler A (1944) Disintegration and restoration of optic recognition in visual agnosia: analysis of a case. Arch Neurol Psychiatry 51:243-259.

Adler A (1950) Course and outcome of visual agnosia. J Nerv Ment Dis 111:41-51.

Barton JJS, Cherkasova MV, Press DZ, Intriligator JM, O’Connor M (2004) Perceptual functions in prosopagnosia. Perception 33:939-956.

Benson DF, Greenberg JP (1968) Visual form agnosia: a specific defect in visual recognition. Trans Am Neurol Assoc 93:189-191.

Benson DF, Greenberg JP (1969) Visual form agnosia. A specific defect in visual discrimination. Arch Neurol 20:82-89.

Blake A, Brady JM (1992) Computational modelling of hand-eye coordination. Philos Trans R Soc Lond B Biol Sci 337:351-360.

Brant-Zawadzki M, Atkinson D, Detrick M, Bradley WG, Scidmore G (1996) Fluid-attenuated inversion-recovery (FLAIR) for assessment of cerebral infarction: initial clinical experience in 50 patients. Stroke 27:1187-1191.
Campion J, Latto R (1985) Apperceptive agnosia due to carbon monoxide poisoning. An interpretation based on critical band masking from disseminated lesions. Behav Brain Res 15:227-240.

Crawford JR, Garthwaite PH (2005) Testing for suspected impairments and dissociations in single-case studies in neuropsychology: evaluation of alternatives using Monte Carlo simulations and revised tests for dissociations. Neuropsychology 19:318-331.

Crawford JR, Howell DC (1998) Comparing an individual's test score against norms derived from small samples. Clin Neuropsychol 12:482-486.

D’Amico M, Ferrigno G (1992) Comparison between the more recent techniques for smoothing and derivative assessment in biomechanics. Med Biol Eng Comput 30:193-204.

Dijkerman HC, Milner AD, Carey DP (1998) Grasping spatial relationships: failure to demonstrate allocentric visual coding in a patient with visual form agnosia. Conscious Cogn 7:424-437.

Downing PE, Jiang Y, Shuman M, Kanwisher N (2001) A cortical area selective for visual processing of the human body. Science 293:2470-2473.

Durak AC, Coskun A, Yikilmaz A, Erdogan F, Mavili E, Guven M (2005) Magnetic resonance imaging findings in chronic carbon monoxide intoxication. Acta Radiol 46:322-327.

Efron R (1968) What is perception? In: Boston studies in the philosophy of science, Vol 4 (Cohen RS, Wartofsky M, eds), pp 137-173. New York: Humanities.

Epstein R, Kanwisher N (1998) A cortical representation of the local visual environment. Nature 392:598-601.

Gauthier I, Skudlarski P, Gore JC, Anderson AW (2000) Expertise for cars and birds recruits brain areas involved in face recognition. Nat Neurosci 3:191-197.

Goodale MA, Milner AD, Jakobson LS, Carey DP (1991) A neurological dissociation between perceiving objects and grasping them. Nature 349:154-156.

Goodale MA, Meenan JP, Bülthoff HH, Nicolle DA, Murphy KJ, Racicot CI (1994) Separate neural pathways for the visual analysis of object shape in perception and prehension. Curr Biol 4:604-610.

Grill-Spector K, Knouf N, Kanwisher N (2004) The fusiform face area subserves face perception, not generic within-category identification. Nat Neurosci 7:555-562.

Hasson U, Levy I, Behrmann M, Hendler T, Malach R (2002) Eccentricity bias as an organizing principle for human high-order object areas. Neuron 34:479-490.

Haxby JV, Gobbini MI, Furey ML, Ishai A, Schouten JL, Pietrini P (2001) Distributed and overlapping representations of faces and objects in ventral temporal cortex. Science 293:2425-2430.

Hsü YK, Ch'eng YL (1938) Cerebral subcortical myelinopathy in carbon monoxide poisoning. Brain 61:384-392.

Iberall T, Bingham G, Arbib MA (1986) Opposition space as a structuring concept for the analysis of skilled hand movements. In: Generation and modulation of action patterns. Experimental brain research series, Vol 15, pp 158-173. Berlin: Springer.

James TW, Culham J, Humphrey GK, Milner AD, Goodale MA (2003) Ventral occipital lesions impair object recognition but not object-directed grasping: an fMRI study. Brain 126:2463-2475.

Kanwisher N (2000) Domain specificity in face perception. Nat Neurosci 3:759-763.

Kanwisher N, Chun MM, McDermott J, Ledden PJ (1996) Functional imagining of human visual recognition. Brain Res Cogn Brain Res 5:55-67.

Kanwisher N, McDermott J, Chun MM (1997) The fusiform face area: a module in human extrastriate cortex specialized for face perception. J Neurosci 17:4302-4311.

Karnath H-O, Perenin M-T (2005) Cortical control of visually guided reaching: evidence from patients with optic ataxia. Cereb Cortex 15:1561-1569.

Kessler J, Schaaf A, Mielke R (1993) Der Fragmentierte Bildertest. Göttingen: Hogrefe.

Kim JH, Chang KH, Song IC, Kim KH, Kwon BJ, Kim HC, Kim JH, Han MH (2003) Delayed encephalopathy of acute carbon monoxide intoxication: diffusivity of cerebral white matter lesions. AJNR Am J Neuroradiol 24:1592-1597.

Konen CS, Kastner S (2008) Two hierarchically organized neural systems for object information in human visual cortex. Nat Neurosci 11:224-231. 
Landis T, Graves R, Benson DF, Hebben N (1982) Visual recognition through kinaesthetic mediation. Psychol Med 12:515-531.

Lapresle J, Fardeau M (1967) The central nervous system and carbon monoxide poisoning. II. Anatomical study of brain lesions following intoxication with carbon monoxide (22 cases). Prog Brain Res 24:31-74.

Levy I, Hasson U, Avidan G, Hendler T, Malach R (2001) Center-periphery organization of human object areas. Nat Neurosci 4:533-539.

Lo CP, Chen SY, Lee K-W, Chen W-L, Chen C-Y, Hsueh C-J, Huang G-S (2007) Brain injury after acute carbon monoxide poisoning: early and late complications. AJR Am J Roentgenol 189:W205-W211.

Malach R, Reppas JB, Benson RR, Kwong KK, Jiang H, Kennedy WA, Ledden PJ, Brady TJ, Rosen BR, Tootell RB (1995) Object-related activity revealed by functional magnetic resonance imaging in human occipital cortex. Proc Natl Acad Sci U S A 92:8135-8139.

McCandliss BD, Cohen L, Dehaene S (2003) The visual word form area: expertise for reading in the fusiform gyrus. Trends Cogn Sci 7:293-299.

McIntosh RD, Dijkerman HC, Mon-Williams M, Milner AD (2004) Grasping what is graspable: evidence from visual form agnosia. Cortex 40:695-702.

Milner AD, Goodale MA (1995) The visual brain in action. Oxford: Oxford UP.

Milner AD, Perrett DI, Johnston RS, Benson PJ, Jordan TR, Heeley DW, Bettucci D, Mortara F, Mutani R, Terazzi E, Davidson DLW (1991) Perception and action in 'visual form agnosia.' Brain 114:405-428.

Mon-Williams M, Tresilian JR, McIntosh RD, Milner AD (2001a) Monocular and binocular distance cues: insights from visual form agnosia I (of III). Exp Brain Res 139:127-136.

Mon-Williams M, McIntosh RD, Milner AD (2001b) Vertical gaze angle as a distance cue for programming reaching: insights from visual form agnosia II (of III). Exp Brain Res 139:137-142.

Morris JC, Heyman A, Mohs RC, Hughes JP, van Belle G, Fillenbaum G, Mellits ED, Clark C (1989) The Consortium to Establish a Registry for Alzheimer's Disease (CERAD). Part I. Clinical and neuropsychological assessment of Alzheimer's disease. Neurology 39:1159-1165.

Noguchi K, Ogawa T, Inugami A, Fujita H, Hatazawa J, Shimosegawa E, Okudera T, Uemura K, Seto H (1997) MRI of acute cerebral infarction: a comparison of FLAIR and T2-weighted fast spin-echo imaging. Neuroradiology 39:406-410.

Oswald WD, Fleischmann UM （1997） Das Nürnberger Altersinventar. Göttingen: Hogrefe.
Peelen MV, Downing PE (2005) Selectivity for the human body in the fusiform gyrus. J Neurophysiol 93:603-608.

Puce A, Allison T, Gore JC, McCarthy G (1995) Face-sensitive regions in human extrastriate cortex studied by functional MRI. J Neurophysiol 74:1192-1199.

Ricci PE, Burdette JH, Elster AD, Reboussin DM (1999) A comparison of fast spin-echo, fluid-attenuated inversion-recovery, and diffusionweighted MR imaging in the first 10 days after cerebral infarction. Am J Neuroradiol 20:1535-1542.

Satzger W, Hampel H, Padberg F, Bürger K, Nolde T, Ingrassia G, Engel RR (2001) Zur praktischen Anwendung der CERAD-Testbatterie als neuropsychologisches Demenzscreening. Nervenarzt 72:196-203.

Schenk T (2006) An allocentric rather than perceptual deficit in patient D.F. Nat Neurosci 9:1369-1370.

Schenk T, Milner AD (2006) Concurrent visuomotor behaviour improves form discrimination in a patient with visual form agnosia. Eur J Neurosci 24:1495-1503.

Servos P, Matin L, Goodale MA (1995) Dissociation between two modes of spatial processing by a visual form agnosic. Neuroreport 6:1893-1896.

Sparr SA, Jay M, Drislane FW, Venna N (1991) A historic case of visual agnosia revisited after 40 years. Brain 114:789-800.

Steeves JK, Humphrey GK, Culham JC, Menon RS, Milner AD, Goodale MA (2004) Behavioral and neuroimaging evidence for a contribution of color and texture information to scene classification in a patient with visual form agnosia. J Cogn Neurosci 16:955-965.

Tarr MJ, Gauthier I (2000) FFA: a flexible fusiform area for subordinatelevel visual processing automatized by expertise. Nat Neurosci 3:764-769.

Ungerleider LG, Mishkin M (1982) Two cortical visual systems. In: Analysis of visual behavior (Ingle DJ, Goodale MA, Mansfield RJW, eds), pp 549586. Cambridge, MA: MIT.

Wann JP, Mon-Williams M, McIntosh RD, Smyth M, Milner AD (2001) The role of size and binocular information in guiding reaching: insights from virtual reality and visual form agnosia III (of III). Exp Brain Res 139:143-150.

Westwood DA, Danckert J, Servos P, Goodale MA (2002) Grasping twodimensional images and three-dimensional objects in visual-form agnosia. Exp Brain Res 144:262-267. 